\title{
JOSÉ, UNA MIRADA AL SER: ANÁLISIS DE UNA NARRATIVA DE ENFERMERÍA DE ACUERDO CON EL MODELO DE ADAPTACIÓN DE ROY
}

LE. Francisca Elvira Blanco Enríquez (1)

Dra. Clara Virginia Caro Castillo (2)

Dra. Olga Janneth Gómez Ramírez (3)

Dra. Leticia Cortaza Ramírez (4)

1.- Facultad de Enfermería, Universidad Veracruzana, Campus Minatitlán, Veracruz, México.

2- Facultad de Enfermería, Departamento de Enfermería, Sede Bogotá / Universidad Nacional de Colombia / Bogotá, Colombia / Correo *(autor de correspondencia)

3.-Facultad de Enfermería, Departamento de Enfermería, Sede Bogotá. Universidad Nacional de Colombia. Bogotá, Colombia.

4.-Facultad de Enfermería, Universidad Veracruzana, Campus Minatitlán, Veracruz, México.

Recibido: 18 de septiembre de 2018

Aceptado: 24 de septiembre de 2018 


\title{
Resumen:
}

La narrativa constituye una expresión real de lo que es la práctica de Enfermería, como una metodología dentro de un nuevo paradigma para la investigación. Con el método de 10 pasos de Smith \& Liehr, se analizó una Narrativa de Enfermería de acuerdo con el Modelo de Adaptación de Callista Roy, permitiendo encontrar significado a la situación al plantear nombre del fenómeno, lente teórico, cualidades esenciales o atributos, una definición esencial y un gráfico que muestra la relación entre las cualidades. Este análisis permitió comprender el fenómeno desde una perspectiva teórica enfermera y comenzar un proceso de generación de teoría.

Palabras clave: Narrativa, Enfermería, Adaptación.

\begin{abstract}
:
The narrative is a real expression of what is the practice of Nursing, as a methodology within a new paradigm for research. With the method of 10 steps of Smith \& Liehr, a Nursing Narrative was analyzed according to the Callista Roy Adaptation Model, allowing to find meaning to the situation proposing name of the phenomenon, theoretical lens, essential qualities or attributes, a definition essential and a graph that shows the relationship between qualities. This analysis allowed to understand the phenomenon from a theoretical nurse perspective and to begin a process of theory generation.
\end{abstract}

Key Words: Narrative, Nursing, Adaptation.

\section{Introducción}

Actualmente, existen múltiples referentes teóricos de Enfermería que instan la utilización de nuevos métodos para la investigación, uno de ellos es la narrativa 
de Enfermería (1); son formas escritas de experiencias reales vividas por los enfermeros, que destacan los aspectos más significativos de su práctica, estas tienen la intención en muchos casos de mostrar el patrón de conocimiento estético como representación del arte de enfermería (2).

El investigador tiene un gran reto de utilizar las narrativas para comprender las experiencias que otros viven. A su vez, a partir de los relatos, se trata de establecer la estructura de un episodio, organizar la secuencia de eventos, explicar a través de la interpretación de los hechos y significados que dan sentido a la experiencia (3).

La aplicación de una narrativa como fuente de conocimiento, permite relacionar la intervención enfermera de la vida diaria con una teoría o modelo de Enfermería, que amplíe la visión del panorama de cuidado al argumentar una situación problema con las cualidades o atributos de un fenómeno, para formular propuestas de cuidado que resulten de los problemas reales de salud.

Smith \& Liehr sugieren un método de diez pasos para crear teoría desde una historia que capte el fenómeno y la posibilidad de darle nombre, confirmando así que una narrativa puede ser fuente para identificar un fenómeno. Con la escalera de abstracción como una estructura propuesta por las autoras, la narrativa es un elemento primario para crear conocimiento y se ubica en el nivel empírico ${ }^{(4 ; 2) .}$

El uso del Modelo de Adaptación de Callista Roy ante el consumo de alcohol en adolescentes, resulta un planteamiento que se relaciona con este fenómeno, por lo que su análisis resulta de suma importancia para la mejora de la salud del joven, su familia y comunidad.

El consumo de alcohol en los adolescentes es una problemática que afecta a la sociedad mundial, el uso abusivo de esta sustancia es de alto riesgo para el origen de una adicción en la adultez. En las Américas, se consume más alcohol que en el resto del mundo. En el caso de los adolescentes, estos beben con menor frecuencia, pero ingieren más cantidad en cada ocasión. En el 2010, alrededor de 14,000 defunciones de menores de 19 años fueron atribuidas al alcohol (5). 
La familia se puede convertir en un factor de riesgo frente al consumo de sustancias cuando hay un desequilibrio en el hogar (6). Asimismo, uno de los factores más comúnmente asociados con el consumo del alcohol es la personalidad, la impulsividad, la búsqueda de sensaciones, el autoconcepto y la conducta antisocial (7).

A medida que pasan los años, se va formando un autoconcepto cada vez más estable y con mayor capacidad para dirigir nuestra conducta, el desarrollo de este puede ser visto desde un enfoque interaccionista: el ambiente posibilita ciertas experiencias las cuales serán tratadas según las posibilidades evolutivas (8). Investigaciones han referido relaciones positivas entre el autoconcepto físico y el consumo de alcohol en adolescentes. También se han encontrado relaciones positivas entre el autoconcepto social, el dominio del autoconcepto, amistad íntima y el consumo de sustancias y relaciones negativas ${ }^{(9)}$.

Un estudio realizado en adolescentes españoles, reportó relaciones negativas entre el autoconcepto familiar, académico y físico, y el consumo de sustancias (10). En otra investigación en una población parecida, indicaron que los datos del autoconcepto académico, social y familiar mostraron relación estadísticamente significativa en todos los casos de ingesta de alcohol (11).

En este sentido, es común encontrar diversos casos problema relacionados con el uso indebido de alcohol adolescente, por lo que las situaciones vividas en las instituciones educativas o de salud que son presenciadas por el profesional de Enfermería son de suma importancia para el análisis de fenómenos que pueden ser atendidos por el personal enfermero y así mejorar el cuidado.

En el presente trabajo, se incluye una narrativa de un adolescente consumidor de alcohol, donde a partir de lo propuesto en el Modelo de Adaptación de Roy, es posible relacionar algunos conceptos del modelo, principalmente el modo de adaptación de autoconcepto, el cual de acuerdo con la literatura científica, es uno de los elementos involucrados en el fenómeno de consumo de alcohol, por lo que se analizan sus atributos y su relación con la situación, que marcan la pauta para el análisis del cuidado respecto a las creencias y pensamientos del joven. 


\section{Narrativa de Enfermería. “José, una mirada al ser”}

Al realizar mi tesis de pregrado, me fue designado un tema de consumo de alcohol en adolescentes, con el paso del tiempo fui reflexionando sobre las consecuencias en la sociedad mundial y cómo Enfermería contribuía con el cuidado de la salud mental; sin embargo, algo de lo que analizaba constantemente eran los problemas que frecuentemente se presentaban entre las personas que me rodeaban e incluso en otras que no eran tan cercanas.

Resultaba algo tan común, que la gran mayoría de compañeros y conocidos contaban con algún familiar alcohólico o conocían a alguien que tenía problemas con su ingesta, lo que aumentó mi sensibilidad ante la problemática actual relacionada con el tema.

Fue así, que mi primer contacto con adolescentes de una comunidad rural, resultó una visita difícil de olvidar, al darme cuenta de lo frecuente que son los problemas relacionados con el consumo de alcohol y los daños que puede causar no sólo en el consumidor, sino en quienes le rodean.

Era una preparatoria de comunidad rural, a la cual acudí para la aplicación de instrumentos sobre prevalencias de consumo de alcohol en adolescentes, me presenté a la institución con una compañera que conocía a gran parte de los residentes de la localidad, por lo que cuando me encontré sola en determinado momento, se me acercó un joven a preguntarme sobre alternativas para disminuir el consumo, mencionaba que le daba pena comentar su caso debido a que mi compañera lo conocía.

Por ello, nos sentamos a platicar en la cancha central apartados de las demás personas, en un ambiente relajado debido a que los estudiantes en ese momento únicamente acudían a la escuela para recibir calificaciones y ya no tenían pendientes 
clases o actividades extras, por lo que se disponía de tiempo para aplicar las encuestas y realizar algunas pláticas informativas.

En ese momento, me comentó de forma nerviosa sobre su situación familiar y personal: “Mi nombre es José, tengo 16 años, soy hijo único, mi padre es alcohólico y es violento con mi mamá, él le pega y yo por defenderla me golpea a mí también", "casi siempre después de clases en la tarde y parte de la noche me voy de la casa con los vecinos o amigos de la escuela a jugar fútbol, en ocasiones bebemos alcohol en calles solitarias después de los partidos o salimos a tomar para platicar".

Por otra parte, José refirió tener novia en la escuela, aunque tenía problemas con ella relacionados con agresividad, pero se relajaba pasando mucho tiempo con sus amigos, aunque con vergüenza que estos fueran a su casa y vieran la situación por la que estaba pasando con su padre alcohólico.

Además, indicó sentirse apenado por pertenecer a este grupo de amigos donde consumen alcohol constantemente, llegando a la embriaguez cada fin de semana, dudaba de sí mismo como persona íntegra y de bien, por lo que comentó: “ me gusta el fútbol, pero pienso que cada vez tomo más y me deprimo, quizás lo hago para olvidar mis problemas en casa o con mi novia", "me siento solo, las personas con las que salgo son compañeros de borrachera, quisiera encontrar a alguien que realmente me aconsejara para bien, ya que en casa no se puede".

Por esto, me acerqué un poco más a su lado para escuchar mejor lo que trataba de contarme, quería que el se sintiera acompañado y que alguien lo apoyaba para salir adelante y no repetir el caso de su padre y maltratar a su familia e incluso a otros seres cercanos.

Le comenté que uno de los factores protectores para evitar la adicción a las drogas y disminuir el consumo de alcohol era realizar deporte, aunque las actividades que llevaba a cabo después de este dependían en gran parte de su persona, al no dejarse llevar por la presión de grupo y participar en las reuniones con conductas de riesgo para la salud que pueden afectar su desarrollo y adultez en un futuro. 
José me respondió con tristeza: "me siento menos que los demás por mi situación en casa, y aún lucho ante mí mismo para calmar mi agresividad con mi novia y otras personas, quizás podría incluirme en otras actividades de la escuela que tengan que ver con los deportes, y tratar de hablar con mi padre y madre para buscar una solución a los problemas".

Ante su respuesta, coloqué mi mano en su hombro comentándole que admiro su valentía de expresar sus sentimientos por la situación que se encuentra viviendo, por lo que destaqué la importancia de contar con apoyo profesional y le invité a asistir a las reuniones y pláticas que realiza la escuela para prevenir adicciones y atender la salud de los estudiantes, que contaba conmigo para platicar y desahogar sus penas.

Al finalizar estas palabras, José me dijo: "gracias por la plática, me hizo bien liberarme un poco de todo lo que ocurre", reiteró su lucha para hablar con sus padres y realizar actividades deportivas sin relacionarse demasiado con el alcohol, se retiró al baño indicándome el salón donde se encuentra su grupo y se despidió.

Sé que la plática con José pudo no haberle ayudado de mucho, pero recordé que sacar todo lo malo que uno trae dentro resulta reconfortante para cualquiera, muchas veces miramos las alteraciones físicas del ser humano, sin cuidar el yo interno que afecta sus creencias personales para la vida.

\section{LA NARRATIVA DE ENFERMERÍA Y SU RELACIÓN CON EL MODELO DE ADAPTACIÓN DE ROY}

De acuerdo con los pasos propuestos por Smith \& Liehr para crear teoría desde una historia, se le otorgó como nombre al fenómeno: “Autoconcepto en adolescentes consumidores de alcohol". Se presenta el lente teórico a través de los tres niveles que representan cada peldaño de la escalera de abstracción, con el aspecto filosófico, 
teórico y empírico, relacionado con el Modelo de Adaptación de Sor Callista Roy y la narrativa de Enfermería antes presentada.

Finalmente, se abordan las cualidades esenciales o atributos del fenómeno, una definición que integra lo esencial del fenómeno, y un gráfico o modelo que muestra la relación entre las cualidades fundamentales.

Nivel filosófico

Las visiones del mundo de enfermería tienen que ver directamente con la filosofía del conocimiento que se considera importante para el desarrollo disciplinar. Las visiones del mundo señaladas por Fawcett, son la de reacción, de interacción recíproca y de acción simultánea ${ }^{(2 ; 12) .}$

En el caso del Modelo de Adaptación de Roy, se relaciona con la visión de interacción recíproca, donde se considera a los seres humanos holísticos, activos e interactivos con su ambiente respondiendo a las interacciones, se ve la realidad como multidimensional, dependiente del contexto y condiciones circundantes, y la realidad es relativa ${ }^{(2 ; 12)}$.

Cabe mencionar, que Sor Callista unió los supuestos del humanismo y de la verdad en un mismo conjunto de supuestos filosóficos de acuerdo con las características ofrecidas por Swimme y Berry de la creación de la espiritualidad, por lo que planteó que las personas se relacionan a la vez con el mundo y con Dios. A su vez, propuso como supuesto que en los últimos tiempos, se ha descubierto a Dios en la diversidad de la creación, y éste constituye el destino común de la creación (13).

Asimismo, Roy está de acuerdo con los teóricos que creen que los cambios que se producen en los sistemas de la persona y de su entorno en el mundo son tan enormes que un gran hito está llegando a su fin. Así como de lo sugerido por Sullivan de que la personalidad de un individuo se forma por medio de la interacción social (13).

Por otro lado, es importante destacar que el concepto central del modelo es la Adaptación, definida como el proceso y resultado por medio de los cuales las personas, que tienen la capacidad de pensar y de sentir, como individuos o como 
miembros de un grupo, son conscientes y escogen la integración del ser humano con su entorno $(14 ; 15)$.

Nivel teórico

El modelo de Roy se centra en el concepto de adaptación de la persona, quien busca continuamente estímulos en su entorno. Como paso último, se efectúa la reacción y se da paso a la adaptación. Esta reacción puede ser eficaz o puede no serlo (14;13).

La meta de Enfermería es la promoción de la adaptación del sistema humano. La adaptación busca mantener la integridad y la dignidad; contribuye a promover, mantener y mejorar la salud, la calidad de vida, y a morir con dignidad (14;16).

De acuerdo con el sistema vital abierto en el Modelo de Roy, la persona recibe estímulos tanto del ambiente como de sí misma. El nivel de adaptación está determinado por el conjunto de efectos que producen los estímulos focales, contextuales y residuales $(14 ; 13)$.

En el Modelo hay dos subsistemas que se relacionan entre sí: el subsistema de procesos primarios, funcionales o de control está compuesto por el regulador y el relacionador; el subsistema secundario y de efectos está compuesto por cuatro modos de adaptación:

a) fisiológico;

b) autoconcepto;

c) función del rol, y

d) interdependencia ${ }^{(14 ; 13)}$.

Roy considera que tanto el regulador como el relacionador son métodos de afrontamiento. El subsistema del regulador, por medio del modo de adaptación fisiológico, «reacciona automáticamente mediante procesos de afrontamiento neuronales, químicos y endocrinos». El subsistema del relacionador, gracias a los modos de adaptación de la autoconcepto, de la interdependencia y de la función del rol, «reacciona por medio de los canales cognitivos y emocionales: el procesamiento de la información sensorial, el aprendizaje, el criterio y las emociones» (14; 13). 
El modo de adaptación de autoconcepto, es definido como «el conjunto de creencias y sentimientos que un individuo tiene de sí mismo en un momento determinado». El autoconcepto individual se compone del yo físico (el aspecto físico y la imagen del cuerpo) y del yo personal (la coherencia y el ideal de uno mismo, y la identidad moral, ética y espiritual) $(14 ; 13)$.

De acuerdo a lo anterior, los conceptos de Enfermería, meta de Enfermería, entorno, modo de adaptación de autoconcepto y autoconcepto individual del Modelo de Adaptación de Roy, se pueden relacionar con la Narrativa de Enfermería (Tabla 1).

Tabla 1. Conceptos del Modelo de Adaptación de Roy y su relación con la Narrativa de Enfermería

\begin{tabular}{|l|l|}
\hline Modelo conceptual & Narrativa de Enfermería \\
\hline Enfermería es la profesión que se & "Era una preparatoria de comunidad rural, a la cual acudí para \\
dedica a la atención sanitaria, y que & la aplicación de instrumentos sobre prevalencias de consumo \\
se centra en los procesos humanos & de alcohol en adolescentes...se me acercó un joven a \\
vitales y en los modelos que se han & preguntarme sobre alternativas para disminuir el consumo, \\
de seguir, y le da importancia a la & mencionaba que le daba pena comentar su caso debido a que \\
promoción de la salud de los & mi compañera lo conocía... nos sentamos a platicar en la \\
individuos, de las familias, de los & cancha central apartados de las demás personas... se disponía \\
grupos y de la sociedad en general & de tiempo para aplicar las encuestas y realizar algunas pláticas \\
(14;15). & informativas".
\end{tabular}




\begin{tabular}{|c|c|}
\hline $\begin{array}{l}\text { La meta de Enfermería es la } \\
\text { promoción de la adaptación del } \\
\text { sistema humano. La adaptación } \\
\text { busca mantener la integridad y la } \\
\text { dignidad; contribuye a promover, } \\
\text { mantener y mejorar la salud, la } \\
\text { calidad de vida, y a morir con }\end{array}$ & $\begin{array}{l}\text { "me acerqué un poco más a su lado para escuchar mejor lo que } \\
\text { trataba de contarme, quería que él se sintiera acompañado y } \\
\text { que alguien lo apoyaba para salir adelante y no repetir el caso } \\
\text { de su padre y maltratar a su familia e incluso a otros seres } \\
\text { cercanos”. } \\
\text { “...le comenté que uno de los factores protectores para evitar } \\
\text { la adicción a las drogas y disminuir el consumo de alcohol era } \\
\text { realizar deporte, aunque las actividades que llevaba a cabo } \\
\text { después de este dependían en gran parte de su persona, al no } \\
\text { dejarse llevar por la presión de grupo y participar en las } \\
\text { reuniones con conductas de riesgo para la salud que pueden } \\
\text { afectar su desarrollo y adultez en un futuro". } \\
\text { "coloqué mi mano en su hombro comentándole que admiraba } \\
\text { su valentía de expresar sus sentimientos por la situación que } \\
\text { se encuentra viviendo, por lo que destaqué la importancia de } \\
\text { contar con apoyo profesional y le invité a asistir a las } \\
\text { todo lo que ocurre” } \\
\text { reuniones y pláticas que realiza la escuela para prevenir } \\
\text { adiones y atender la salud de los estudiantes, que contaba }\end{array}$ \\
\hline
\end{tabular}




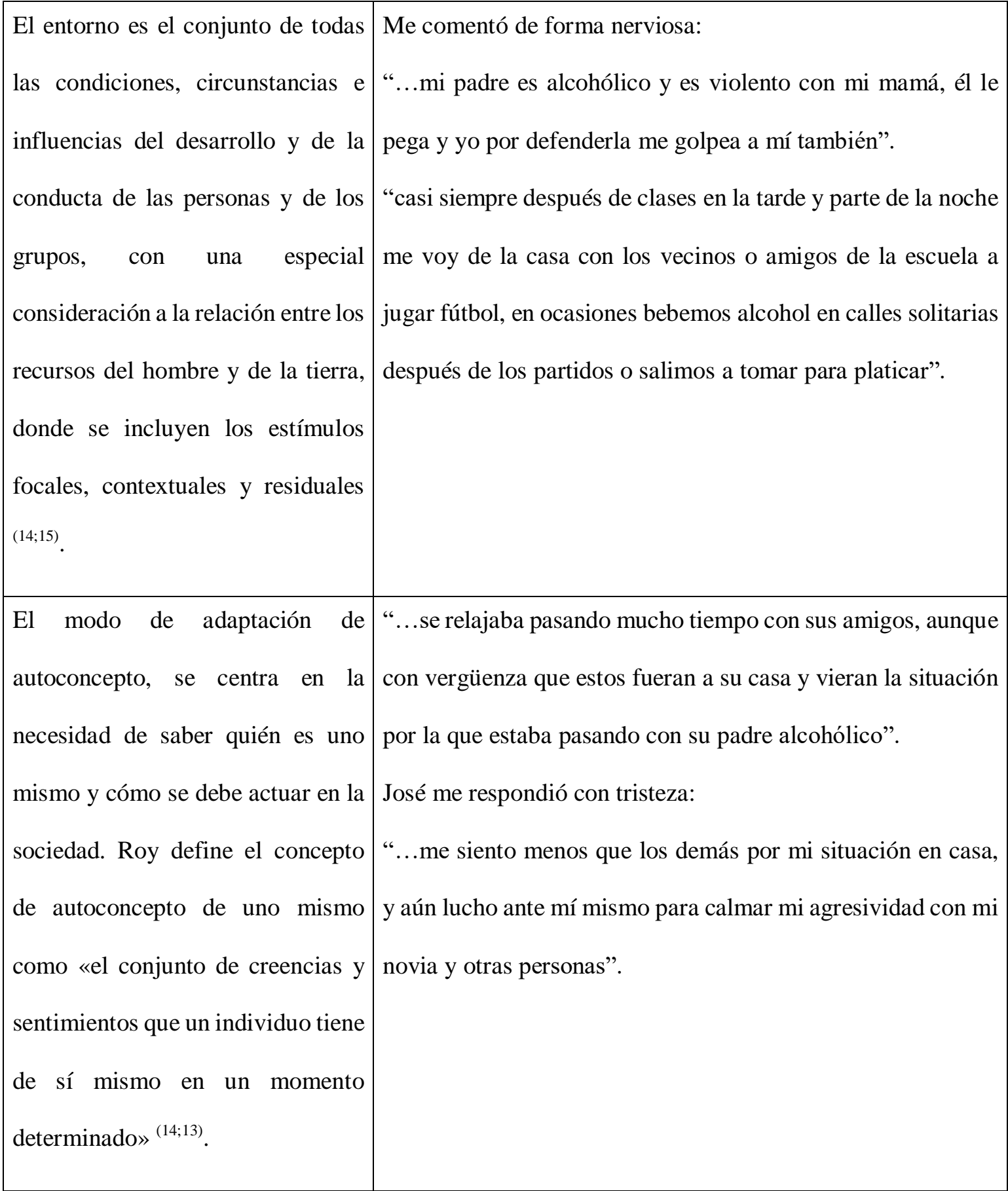




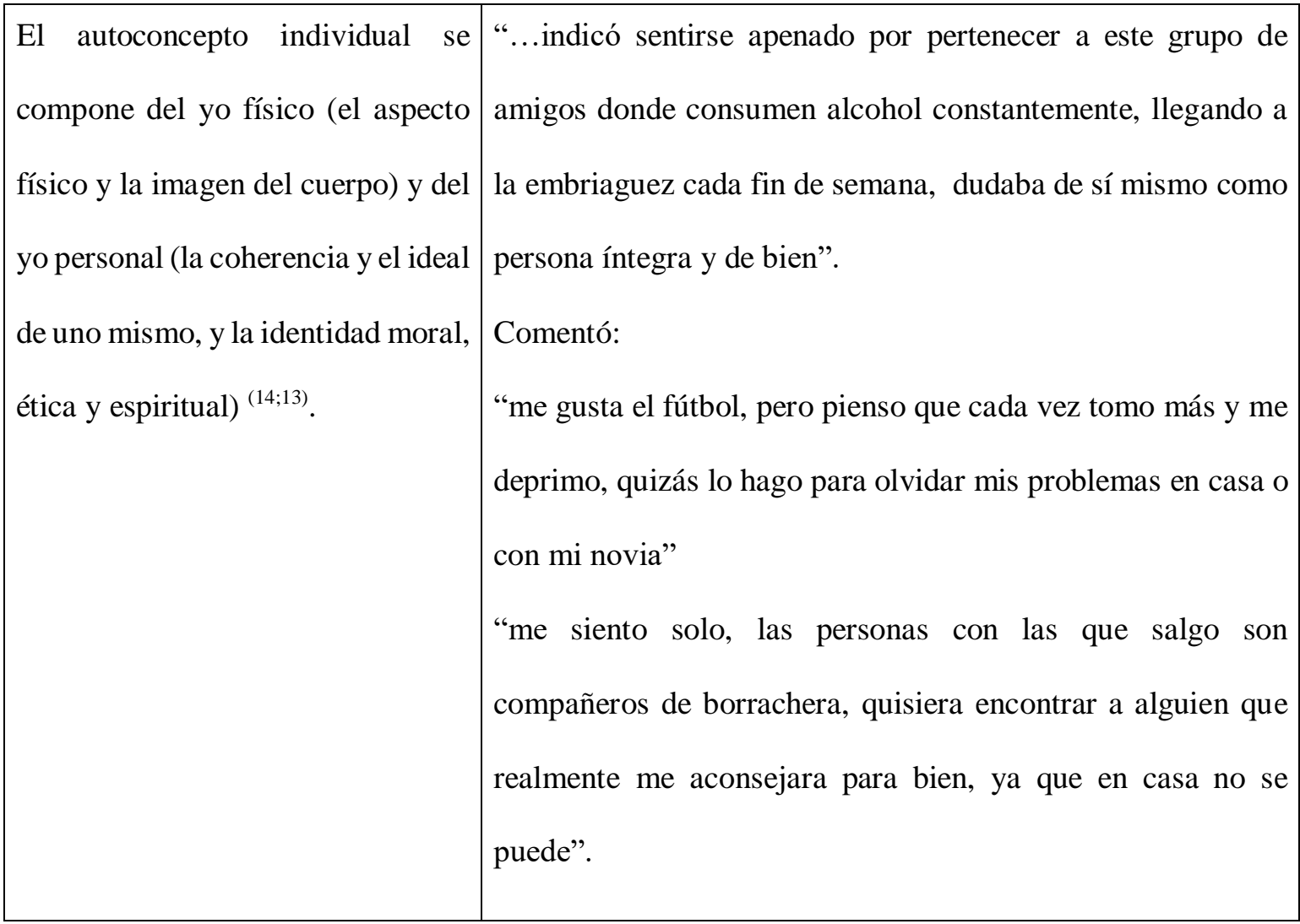

Fuente: Elaboración propia de los autores.

\section{Nivel empírico}

El Modelo de Adaptación de Roy está profundamente enraizado en la práctica enfermera y esto, en parte, contribuye a su éxito continuado. Sigue siendo uno de los marcos conceptuales utilizado con mayor frecuencia y se usa nacional e internacionalmente. Además, el modelo sigue generando gran cantidad de hipótesis comprobables que deben investigarse. El modelo de adaptación de Roy ha sido ampliamente utilizado para guiar el desarrollo del conocimiento a través de la investigación enfermera(13).

Uno de los instrumentos utilizados entre la comunidad científica para medir el Autoconcepto de acuerdo a lo propuesto por Roy es la Escala de Tennessee realizada por Fitts en 1965. Es un instrumento eficaz que incluye la forma de pensar, cómo se ven, valoran y describen a sí mismos las personas, además de la forma en que creen que la sociedad los percibe, es una escala de valoración auto-aplicable (17). 
La escala está compuesta por 100 ítems/preguntas. La dimensión interna consta de tres escalas:

a) Identidad o autoconcepto;

b) Autosatisfacción o autoestima; y

c) Autocomportamiento. Mientras la dimensión externa consta de cinco escalas o factores: Personal, Familiar, Moral-ético, Social y Físico (18).

En México, la escala fue adaptada por Álvarez y Barretos, en 1969, en el estado de Chihuahua, en pacientes con Diabetes Mellitus ${ }^{(17}$ ).

El analizar este nivel empírico, así como los niveles filosófico y teórico, contribuye a pensar en ideas de investigación, al ir de un fenómeno a la teoría, por lo que se realizó en forma de figura la escalera de abstracción (Figura 1).

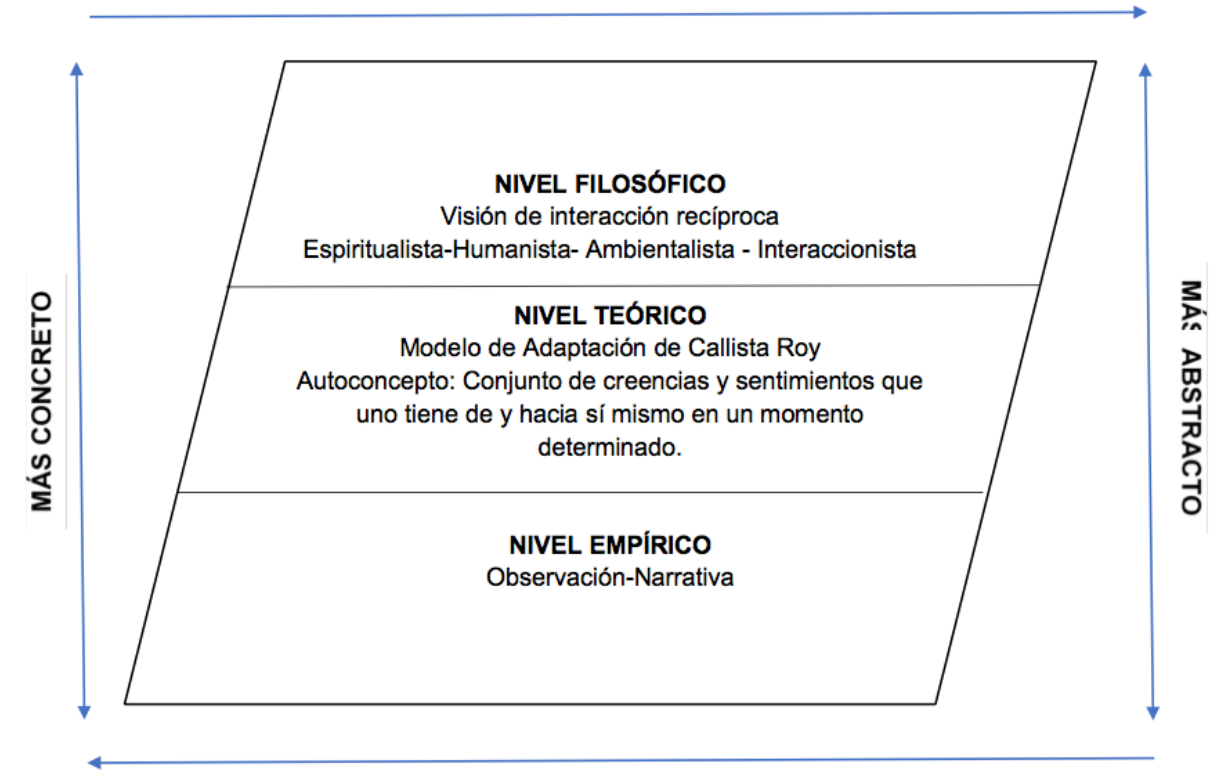

Figura 1. Escalera de abstracción Fuente: Elaboración propia de los autores.

Cualidades esenciales o atributos del fenómeno

El autoconcepto puede definirse como el conjunto de creencias y sentimientos que uno tiene de y hacia sí mismo en un momento determinado. Se forma por la percepción interna y por la percepción de las reacciones de los demás individuos o 
identidad de grupo. El modo de adaptación de Autoconcepto de identidad de grupo refleja "cómo se ve y percibe la gente dentro de un grupo, basándose en reacciones ante el entorno". La necesidad básica de la identidad de grupo es la integridad de la identidad $(14 ; 13)$.

Percepción interna:

Yo físico: engloba las sensaciones y el aspecto del cuerpo.

Yo de la persona: está formado por la constancia, los valores o expectativas, y la moral, la ética y la espiritualidad del ser.

Percepción de identidad de grupo:

Relaciones interpersonales.

Imagen que se tiene del grupo, el entorno social y la cultura.

Definición que integra lo esencial del fenómeno

El autoconcepto del adolescente consumidor de alcohol, lo integran la percepción interna y de identidad de grupo, es decir, las creencias que tiene sobre sí mismo respecto a su persona física, los valores y expectativas ante el uso de la sustancia, así como las ideas propias sobre las relaciones como parte de un grupo social.

Se realizó un gráfico o modelo que muestra la relación entre las cualidades fundamentales y el fenómeno, por lo que se plantearon los atributos en relación al consumo de alcohol.

\section{Conclusiones}


Con la presente situación de Enfermería, se plantea una narrativa aparentemente común entre los adolescentes y familias actuales, la cual involucra al autoconcepto como elemento importante relacionado con el consumo de alcohol. Sin embargo, poco se ha planteado de las experiencias vividas por parte de Enfermería respecto a este fenómeno de estudio, por lo que su análisis y propuesta teórica relacionada con el tema, resulta una relación significativa entre el autoconcepto del adolescente y el consumo de alcohol, ya que se ve afectado el joven, su familia y probablemente la comunidad.

Analizar estos casos de acuerdo con las narrativas de Enfermería, ilustra un panorama del cuidado de Enfermería respecto a la práctica, por lo que su establecimiento permite conocer las situaciones que se presentan en el quehacer diario, las cuales requieren de proyectos e intervenciones que atiendan las necesidades planteadas.

\section{Bibliografía}

1. Ruiz-Bugarin, C. L. (2015). Narrativa de Enfermería: dejando ir a doña paula. ENE Revista de Enfermería, 9(2). doi: 10.4321/S1988-348X2015000200009

2. Gómez-Ramírez, O. J. y Gutiérrez-De Reales, E. (2011). La situación de Enfermería. Editorial Universidad Nacional de Colombia. Bogotá, Colombia.

3. Valderrama-Sanabria, M. L., Peña-Pita, A. P. y Clavijo-Álvarez, L. M. (2017). Narrativa: el estudiante de Enfermería aprendiendo el arte de cuidar. Revista cuidarte, 8(1), 1488-1498.

4. Smith, M. J. \& Liehr, P. R. (2014). Middle Range Theory for Nursing (Third Edition). Springer Publishing Company, LLC. NY, USA. 
5. Organización Panamericana de la Salud (OPS) (2015). Informe sobre la situación regional sobre el alcohol y la salud en las Américas. Consulta 11 de Octubre, 2017, de la Panamerican Health Organization: http://www.paho.org/hq/index.php?option=com_content\&view=article\&id=11 108\%3A2015-regional-report-alcoholhealth\&catid=1893\%3Anews\&Itemid=41530\&lang=es.

6. Méndez-Ruiz, M. D., Armendáriz-García, N. A., Alonso-Castillo, M. M. y LimaRodríguez, J. S. (2016). Modelo de adaptación para evitar el consumo de alcohol en adolescentes de padres alcohólicos. Revista Nure, 13(82), 1-9.

7. Gonzálvez, M. T., Espada, J. P., Guillén-Riquelme, G., Secades, R. y Orgilés, M. (2016). Asociación entre rasgos de personalidad y consumo de sustancias en adolescentes españoles. Revista Adicciones, 28(2), 108-115.

8. Luna-Cazalla, N. y Molero, D. (2013). Revisión teórica sobre el autoconcepto y su importancia en la adolescencia. Revista Electrónica de Investigación y Docencia (REID), 10, 43-64.

9. Calero, A., Schmidt, V. y Bugallo, L. (2016). Consumo de alcohol y su relación con la autopercepción adolescente. Salud y Drogas, 16(1), 49-58.

10. Fuentes, M. C., García, F., Gracia, E. y Lila, M. (2011). Autoconcepto y consumo de sustancias en la adolescencia. Revista Adicciones, 23(3), 237-248.

11. Álvaro-González, J. I., Zurita-Ortega, F., Castro-Sánchez, M., MartínezMartínez, A. y García-Sánchez, S. (2016). Relación entre consumo de tabaco y alcohol y el autoconcepto en adolescentes españoles. Revista Complutense de Educación, 27(2), 533-550.

12. Bueno-Robles, L. S. (2011). Aspectos ontológicos y epistemológicos de las visiones de enfermería inmersas en el quehacer profesional. Ciencia y Enfermería, $17(1), 37-43$.

13. Raile-Alligoog, M. (2014). Modelos y teorías en Enfermería (8va. Edición). Editorial Elsevier. Barcelona, España. 
14. Roy, C. (2009). The Roy Adaptation Model (Third Edition). Pearson Education. New Jersey, USA.

15. Andrade-Cepeda, R. M. G. y López-España, J. T. (2012). Proceso de atención de Enfermería: Guía interactiva para la enseñanza. Editorial Trillas. México D.F.

16. Díaz de Flores, L., Durán de Villalobos, M. M., Gallego-De Pardo, P., GómezDaza, B., Gómez-De Obando, E., González-De Acuña, Y., Gutiérrez-De Giraldo, M. C., Hernández-Posada, A., Londoño-Maya, J. C., Moreno-Fergusson, M. E., PérezGiraldo, B., Rodríguez-Carranza, C., Rozo-De Arévalo, C., Umaña-De Lozano, C., Valbuena, S., Vargas-Márquez, R. \& Venegas-Bustos, B. C. (2002). Análisis de los conceptos del modelo de adaptación de Callista Roy. Aquichan, 2(1), 19-23.

17. Díaz-Oviedo, A. (2014). Diabetes Mellitus: un estudio sobre el nivel de adaptación fisiológica y de autoconcepto en pacientes que participan en un grupo de apoyo. Revista electrónica sobre Cuerpos académicos y Grupos de investigación en Iberoamérica, 1(2).

18. Chacón-López, H. y Lopéz-Justicia, D. (2016). Relación entre autoconcepto y nivel de depresión en personas con retinosis pigmentaria. Anales de Psicología, 32(3), 820-827. doi: 10.6018/analesps.32.3.218081 